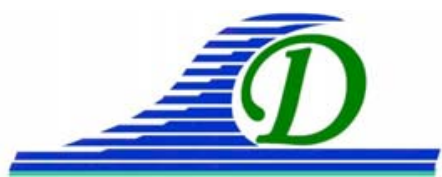

XIII ${ }^{\text {èmes }}$ Journées Nationales Génie Côtier - Génie Civil

Dunkerque, 2-4 juillet 2014

DOI:10.5150/jngcgc.2014.016 @ Editions Paralia CFL

disponible en ligne - http://www.paralia.fr - available online

\title{
Période de retour des évènements de fortes houles le long des côtes françaises (Atlantique et Manche)
}

\section{Alexandre NICOLAE LERMA ${ }^{1}$, Thomas BULTEAU ${ }^{1}$, Sophie LECACHEUX ${ }^{1}$, Déborah IDIER ${ }^{1}$}

1. BRGM, Direction Risques et Prévention, Unité Risques Côtiers et Changement climatique, Orléans, 3 avenue Claude Guillemin, BP36009 - 45060 Orléans, France. a.nicolaelerma@brgm.fr,t.bulteau@brgm.fr, s.lecacheux@brgm.fr,d.idier@brgm.fr

\section{Résumé :}

Les vagues jouent un rôle dominant aussi bien dans l'évolution morphologique des littoraux que sur les phénomènes de submersion. Cet article vise à donner une meilleure connaissance des évènements de forte houle (nombre, période de retour, emprise) à l'échelle pluri-décennale (période janvier 1958 - août 2002). A cet effet, la base de données BoBWA-10kH est analysée pour en déduire des statistiques d'extrêmes le long des côtes atlantique et Manche métropolitaines, permettant ainsi d'estimer la période de retour de hauteurs de vagues observées $(\operatorname{Tr}(\mathrm{Hs}))$. Les évènements majeurs de fortes houles sont présentés et analysés. Ainsi, 7 évènements présentant des hauteurs de houle de période de retour supérieure à 50 ans et affectant les différentes côtes françaises sont identifiées.

L’emprise des évènements sur le linéaire côtier est également observée (extension des conditions de houles $\operatorname{Tr}(\mathrm{Hs})>10$ ans). Les extensions spatiales les plus fortes sont relevées en décembre $1979(\approx 950 \mathrm{~km})$ et février $1989(\approx 650 \mathrm{~km})$. En outre, cette analyse met en évidence des périodes remarquables en termes de succession d'évènements de fortes houles à quelques semaines voire quelques jours d'écart, comme en 1965 et 1989-1990. En termes de caractérisation d'évènements marquants, les périodes de retour des vagues lors des évènements Lothar et Martin sont aussi analysées, mettant en évidence des hauteurs de vagues relativement modérées lors de ces évènements $(\operatorname{Tr}(\mathrm{Hs})<10$ ans). Enfin, l'utilisation de la base de données BoBWA-X est discutée pour l'analyse d'évènements postérieurs à 2002.

Mots-clés : Fortes houles, statistiques d'extrêmes, tempêtes historiques, BoBWA-X.

\section{Introduction}

De nombreuses applications nécessitent des connaissances approfondies des climats de vagues et de leurs caractéristiques (ex: hauteur de vague de période de retour centennale), que ce soit l'ingénierie côtière, le dimensionnement d'ouvrage ou la prévention des risques littoraux. Toutefois, sur les littoraux français, qu'ils soient associés à des conditions locales de tempête, ou à la circulation au large de puissants systèmes dépressionnaires, il existe relativement peu d’informations sur les évènements 


\section{Thème 1 - Hydrodynamique côtière}

passés (échelle de temps pluri-décennale) de fortes houles (dates, intensité, origines, période de retour). Les données d'observation s'avèrent ponctuelles, lacunaires et limitées dans le temps. Les premières mesures côtières au large du littoral métropolitain remontent aux années 1980 et la plupart des bouées constituant le réseau français CANDHIS n’a été mise en place qu’au début des années 2000.

Bien que des conditions de fortes houles à la côte ne soient pas synonymes de dégâts importants, on peut noter que la concomitance de ce type d'évènements avec de forts coefficients de marée et des conditions de surcotes atmosphériques peut provoquer des phénomènes d'érosion et des submersions catastrophiques. Cet article se concentre sur les évènements de fortes houles, indépendamment des phénomènes d'érosion ou submersion.

Actuellement, la base de données ANEMOC (BENOIT et al., 2006), réalisée à partir de simulations rétrospectives de conditions d'état de mer sur la période 1979-2002, est la base de données de référence sur les extrêmes de vagues à l'échelle nationale. LECACHEUX \& PARIS (2013) ont réalisé une étude d'inter-comparaison des données rétrospectives régionales de vagues disponibles. Ils montrent que les données ANEMOC présentent une surestimation des valeurs supérieures au quantile Hs90 (i.e. les $10 \%$ des vagues les plus fortes). ANEMOC propose donc des valeurs extrêmes plutôt sécuritaires, tandis que la base de données rétrospective des conditions d'état de mer BoBWA-10kH (CHARLES et al., 2012, http://bobwa.brgm.fr), couvrant la période janvier 1958 - août 2002, présente les erreurs statistiques les plus faibles par rapport aux observations (ex bouée Gascogne : R2=0,98; RMSE=0,32 m ; SI=0,13). A partir de cette base de données, BULTEAU et al. (2013a, 2013b) ont construit une base de données de valeurs extrêmes de Hs (BoBWA-X), constituée de points d'analyse statistique tout le long du littoral atlantique et de la Manche.

L'objectif de cette étude est d'analyser et cartographier les évènements de fortes houles le long des côtes atlantiques et de la Manche sur la période 1958-2002, en se basant sur l'utilisation conjointe des séries temporelles de vagues (BoBWA-10kH) et de la base de données BoBWA-X fournissant les périodes de retour de hauteurs vagues $(\operatorname{Tr}(\mathrm{Hs}))$. Cet article rappelle tout d'abord la méthode de construction de la base de données de statistiques d'extrêmes BoBWA-X (§2). Les évènements majeurs, tels que leur période de retour $(\operatorname{Tr}(\mathrm{Hs}))$ est supérieure à 10 ans, sont présentés puis leurs intensités et leurs emprises spatiales sont analysées (§3). Enfin, la validité de ces résultats est discutée afin de caractériser les évènements postérieurs à 2002 (§4).

\section{BoBWA-X : description}

BoBWA-X est une base de données de statistiques d'extrêmes de vagues. Les méthodes utilisées pour sa construction, ainsi que les résultats (fiches descriptives, figure 1) sont disponibles dans (BULTEAU et al. 2013a). Nous rappelons ici les éléments principaux servant notre analyse. 


\section{XIII ${ }^{\text {èmes }}$ Journées Nationales Génie Côtier - Génie Civil \\ Dunkerque, 2-4 juillet 2014}

\subsection{Sélection des points d'études}

L'analyse statistique ayant conduit à la constitution de la base de données BoBWA-X a été effectuée sur 43 points répartis le long de la côte atlantique et en Manche (environ tous les $50 \mathrm{~km}$ ) ou co-localisés avec des bouées (figure 1). Ils ont été positionnés en fonction des particularités de la côte à des profondeurs entre $50 \mathrm{~m}$ et $100 \mathrm{~m}$ pour la façade atlantique, et entre 30 et $50 \mathrm{~m}$ pour la Manche. Les données sont de résolution $6 \mathrm{~h}$. Ponctuellement (pour 9 points), les données sont de résolution $6 \mathrm{~h}$ et $1 \mathrm{~h}$. L'analyse des différences sur le paramètre Hs entre les données 1 h et 6 h montre que la différence relative maximale calculée pour la hauteur significative centennale entre les deux séries est de moins de 5\%. Pour notre étude, lorsque les données étaient disponibles, les deux séries de données (1h et $6 \mathrm{~h}$ ) ont été analysées.

L'étude spécifique des épisodes de forte houle à la bouée Gascogne (Golfe de Gascogne) et à la bouée Minquiers (Manche) montre une très bonne reproduction des valeurs de pics lors des évènements de fortes houles $\left(\mathrm{R}^{2} \sim 0,98\right.$; RMSE $=0,47 \mathrm{~m}$, NRMSE $=5,9 \%$ ). Les données issues de BoBWA-10kH sont donc proches des observations et permettent de reconstituer au plus près les évènements ancien de fortes houles.

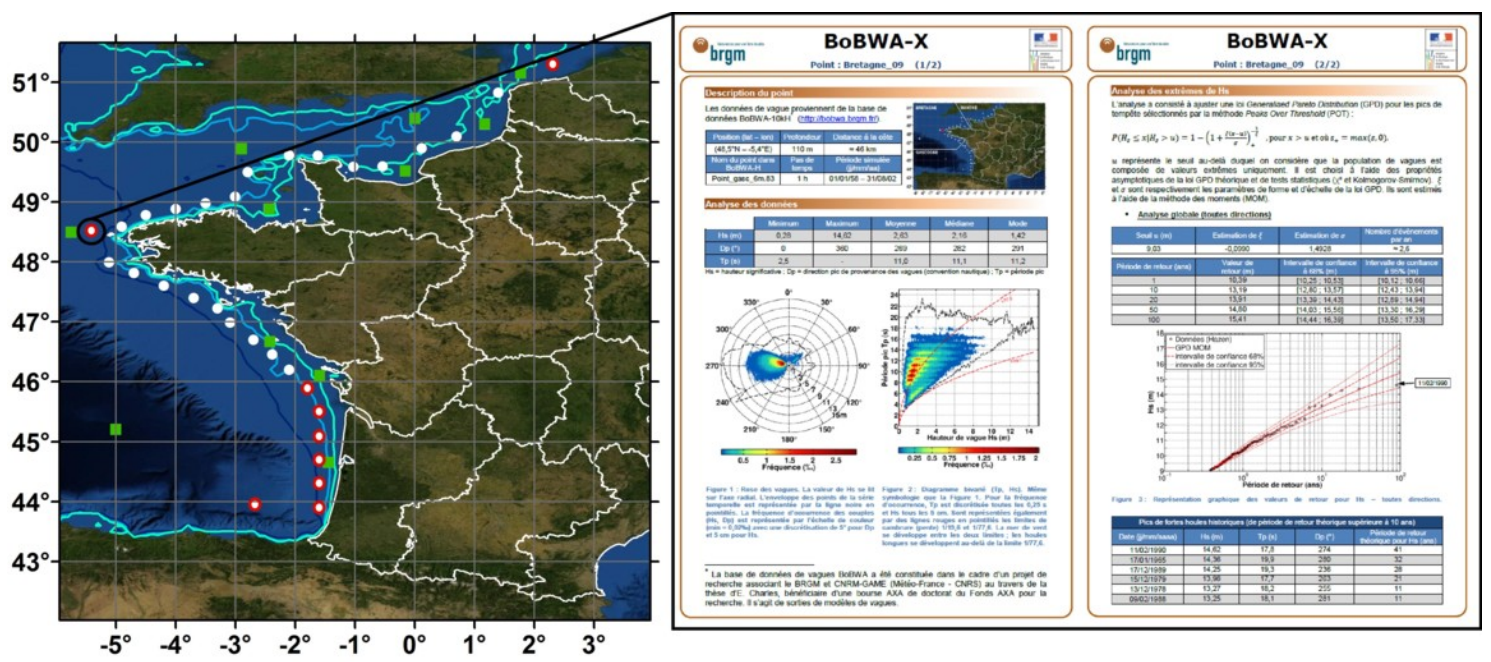

Figure 1. A gauche, localisation des points pour l'analyse statistique : points de grille (ronds) et positions de bouées (carrés), points traités en double avec les données $1 \mathrm{~h}$ et $6 \mathrm{~h}$ (cercles rouge). Les contours correspondent aux isobathes $30 \mathrm{~m}$ (bleu ciel), $50 \mathrm{~m}$ (bleu) et 100m (bleu foncé). A droite, exemple de fiche descriptive de la base de données BoBWA-X.

\subsection{Méthode statistique de calcul des extrêmes}

La méthode de calcul d'extrêmes s'inspire de celle proposée par MAZAS \& HAMM (2011). Il s'agit d'une approche double-seuil de la combinaison classique Peaks-OverThreshold (POT) / Generalized Pareto Distribution (GPD). Basé sur une démarche 


\section{Thème 1 - Hydrodynamique côtière}

itérative combinant tests visuels et statistiques, cette approche permet notamment de réduire au maximum l'incertitude sur les résultats liée au choix, souvent subjectif (THOMPSON et al., 2009), du seuil de la loi GPD et assure ainsi l'homogénéité et une certaine reproductibilité des résultats. Ces qualités associées aux critères de localisation des points (profondeur/exposition aux vagues) a permis de constituer une base de données homogène. La période de retour (en années) des évènements ayant servi à ajuster la loi GPD a été calculée en chaque point tel que : $\operatorname{Tr}=(P(X \geq X) \times n)^{-1}$. P est la probabilité annuelle de dépassement et $\mathrm{n}$ le nombre d'évènement par an.

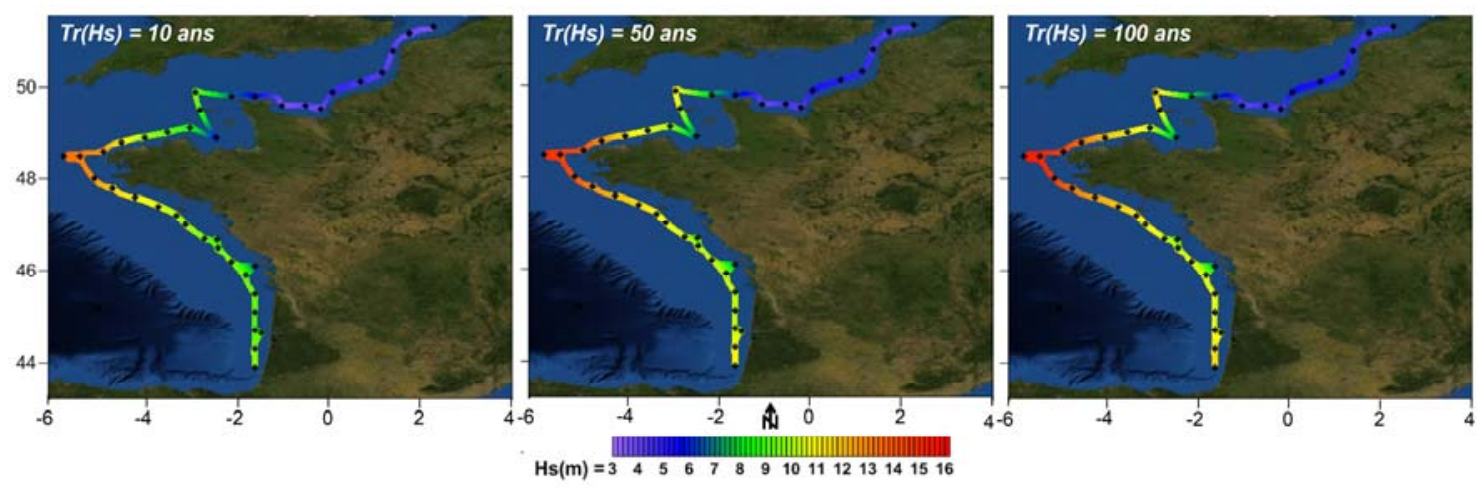

Figure 2. Cartes de synthèse à l'échelle nationale illustrant la variabilité spatiale des valeurs de Hs calculées selon leurs périodes de retour.

\section{Résultats}

\subsection{Les évènements majeurs de fortes houles ( $\operatorname{Tr}>10$ ans)}

A partir de la base de données BoBWA-X fournissant les statistiques d'extrêmes de hauteurs vagues Hs et de la base de données BoBWA-10kH fournissant les séries temporelles de vagues en chaque point d'analyse, les évènements dont les périodes de retour théorique $\operatorname{Tr}(\mathrm{Hs})$ sont supérieurs à 10 ans sont identifiés (tableau 1). On détaille ici la date et l'heure du pic de Hs, l'ordre de grandeur de la période de retour de telles conditions de vague, la ou les régions littorales concernées par les plus fortes période de retour, le département le plus concerné et enfin les régions limitrophes ayant été également concernées par des Hs de périodes de retour supérieurs à 10 ans lors du même évènement.

\subsection{Les évènements majeurs de fortes houles ( $\operatorname{Tr}>50$ ans)}

Sept évènements (E5(1), E5(2), E8, E10, E16, E18, E19, tableau 1) ont été recensés, durant lesquelles les hauteurs de houles simulées ont dépassé une période de retour théorique de 50 ans (cf. figure 3). Chacun de ces évènements a pu être associé à une tempête majeure (en termes de météorologie) au sein des inventaires existants, BESSEMOULIN (2002), MEDD (2002) ou de bulletins météorologiques "MetMar". 


\section{XIII ${ }^{\text {èmes }}$ Journées Nationales Génie Côtier - Génie Civil \\ Dunkerque, 2-4 juillet 2014}

Tableau 1. Synthèse des évènements de fortes houles sélectionnés. $\operatorname{Tr}(\mathrm{Hs})=$ période de retour des hauteurs significatives

\begin{tabular}{|c|c|c|c|c|}
\hline \multirow{2}{*}{$\begin{array}{l}N^{\circ} \text { Evènement et } \\
\text { Date (TU) }\end{array}$} & \multirow{2}{*}{$\begin{array}{l}\text { Heure } \\
(T U)\end{array}$} & \multirow{2}{*}{$\begin{array}{l}\operatorname{Tr} \\
\max ^{*} \\
\text { (ans) } \\
\end{array}$} & \multicolumn{2}{|l|}{ Localisation } \\
\hline & & & Département (Tr max) & Autres régions (Tr>10 ans) \\
\hline E1- 12/07/1959 & $12-13 h$ & $30-50$ & Vendée & Bretagne (sud), Poitou-Charentes \\
\hline$E 2-12 / 04 / 1960$ & $06 h$ & $10-20$ & Pas de Calais & - \\
\hline E3 -01/11/1962 & $12-13 h$ & $10-20$ & Pas de Calais & Picardie \\
\hline$E 4-16 / 12 / 1962$ & $00-01 h$ & $20-30$ & Calvados & H. Normandie \\
\hline E5(1)-17/01/1965 & $18-19 h$ & $50-70$ & Pyrénées Atlantique, Landes & Poitou-Charentes \\
\hline E5(2)-21/01/1965 & $22-00 \mathrm{~h}$ & $70-100$ & Côte d'Armor & B. Normandie \\
\hline E6-21/01/1971 & $18 \mathrm{~h}$ & $20-30$ & Morbihan, Loire Atlantique & - \\
\hline E7-16/01/1974 & $18-22 h$ & $20-30$ & Manche & H. Normandie, Picardie \\
\hline E8-02/12/1976 & $06 h$ & $70-100$ & Gironde & Vendée \\
\hline E9-13/12/1978 & $00-01 h$ & $10-20$ & Finistère & - \\
\hline E10-15/12/1979 & $06-12 h$ & $>100$ & Calvados & Bretagne, H. Normandie, Picardie \\
\hline E11-09/02/1983 & $22 h$ & $10-20$ & Manche & - \\
\hline E12-24/01/1984 & $01 \mathrm{~h}$ & $10-20$ & Manche & - \\
\hline E13-02/07/1984 & $00-01 \mathrm{~h}$ & $20-30$ & Seine Maritime & Basse Normandie \\
\hline E14-16/10/1987 & $06 h$ & $10-20$ & Pas de Calais & - \\
\hline E15-02/09/1988 & $18 \mathrm{~h}$ & $10-20$ & Côte d'Armor & - \\
\hline E16-26/02/1989 & $00-02 h$ & $50-70$ & Landes & Vendée, PdL, Bretagne (sud) \\
\hline E17-17/12/1989 & $06 h$ & $30-50$ & Finistère & - \\
\hline E18-25/01/1990 & $12-18 h$ & $>100$ & Pas de Calais & $\begin{array}{l}\text { Picardie, } H . \text { Normandie, } B . \\
\text { Normandie, Bretagne (Nord) }\end{array}$ \\
\hline E19-12/02/1990 & $00-01 h$ & $70-100$ & Finistère & B. Normandie, $P d L$ \\
\hline E20-28/02/1990 & $18-20 h$ & $20-30$ & Somme & $\begin{array}{l}\text { Nord-Pas de Calais, H. Normandie, } \\
\text { Basse Normandie }\end{array}$ \\
\hline E21-12/08/1993 & $00-01 \mathrm{~h}$ & $10-20$ & Pas de Calais & - \\
\hline E22-02/07/1996 & $18-21 h$ & $10-20$ & Landes & - \\
\hline E23-29/10/1996 & 06h & $10-20$ & Seine Maritime & B. Normandie \\
\hline E24-27/12/1999 & $21-23 h$ & $10-20$ & Landes & - \\
\hline
\end{tabular}

Ces évènements, plus ou moins localisés, ont affecté alternativement l'ensemble des régions de l'Atlantique et de la Manche. Ils indiquent une répartition régionale très nette des évènements de fortes houles. La Bretagne est la région la plus concernée en nombre d'évènements, avec 2 évènements dont la période de retour des vagues est supérieure à 50 ans. Il apparait que les évènements tels que $\operatorname{Tr}(\mathrm{Hs})>50$ ans qui ont affecté les côtes de Vendée et d'Aquitaine, ont engendré des hauteurs de vagues de période de retour modérée en Manche (ex: E16- 26/02/1989, figure 3) et inversement (ex: E1015/12/1979). Ainsi, on n’observe pas, au sein des évènements recensés, de situations où 


\section{Thème 1 - Hydrodynamique côtière}

les conditions de vagues soient observées avec des périodes de retour $\operatorname{Tr}(\mathrm{Hs})$ supérieures à 10 ans simultanément sur les côtes de la Manche (Est du Cotentin) et du Golfe de Gascogne.

Les évènements recensés permettent de mettre en lumière deux types d'informations : l'extension du littoral affecté et la succession d'évènements majeurs.

L'étendue du littoral affectée par des houles de $\operatorname{Tr}(\mathrm{Hs})>10$ ans, lors des événements E10-15/12/1979 et E16-26/02/1989 a été tout à fait remarquable. Ces évènements ont concernés respectivement un linéaire côtier de plus de $950 \mathrm{~km}$ (Manche et Bretagne) et de 650 km (de la Bretagne au Pays Basque). Toutefois ces évènements ne correspondent pas aux situations de $\operatorname{Tr}(\mathrm{Hs})$ les plus fortes.
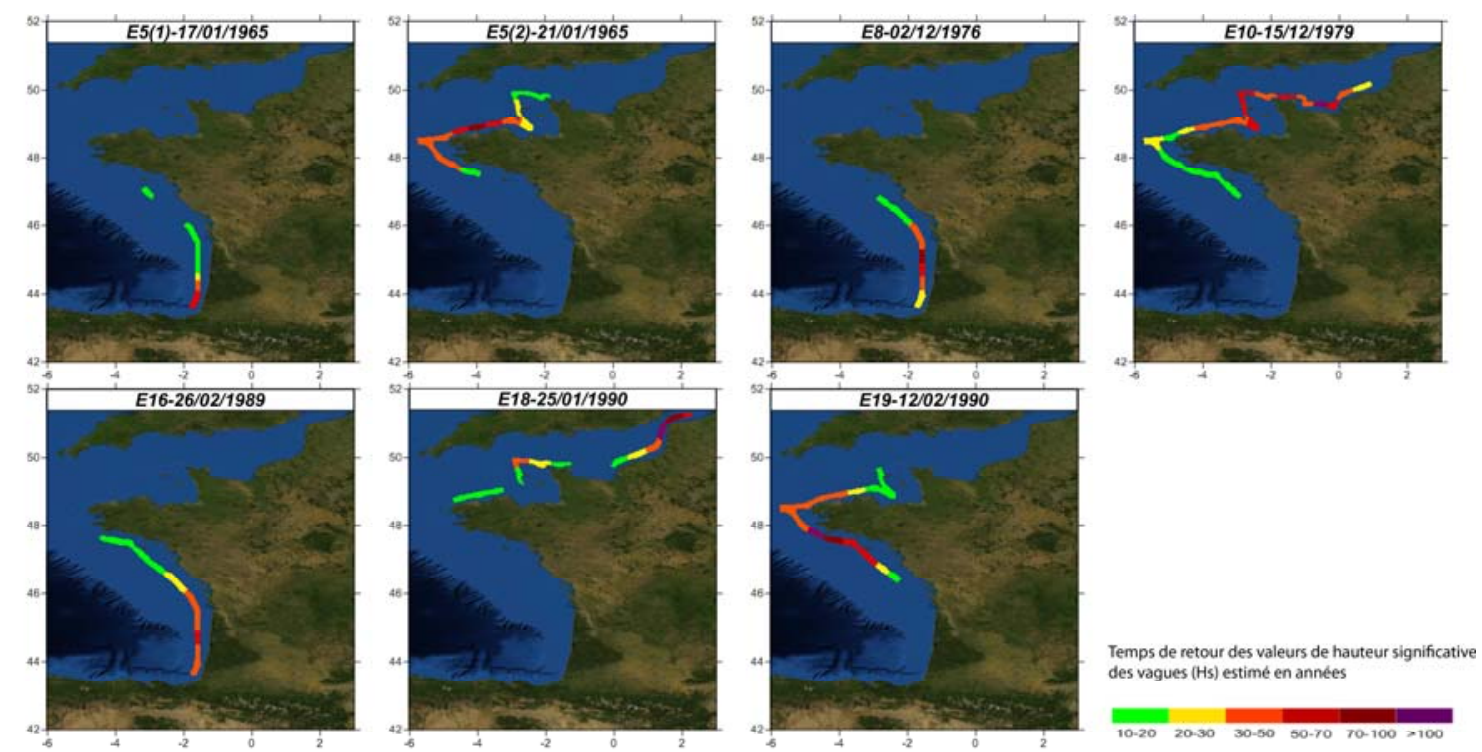

Figure 3. Evènements de fortes houles (période 1958-2001) dont $\operatorname{Tr}(\mathrm{Hs})>50$ ans pour au moins un secteur du littoral et emprise correpondant à $\operatorname{Tr}(\mathrm{Hs})>10$ ans lors du même évenement.

La succession d'évènements majeurs à quelques semaines voir jours d'écart est un second élément marquant. On observe notamment lors de l'année 1965, les conséquences de la circulation de 3 systèmes dépressionnaires consécutifs entre le 13 et le 21 janvier (premier évènement tel que $\operatorname{Tr}(\mathrm{Hs}) \approx 1$ ans, non référencé dans le tableau 1 , et deux suivants tels que $\operatorname{Tr}(\mathrm{Hs})>50$ ans, tableau 1). Durant ces évènements, les houles ont été particulièrement intenses et ont affecté successivement les côtes d'Aquitaine et de Vendée (le 17) puis de Bretagne et de Normandie (le 21). La fin de l'année 1989 et le début de l'année 1990 a été une période de circulations de dépression sur l'Europe du Nord particulièrement active, avec sept tempêtes majeures se succédant du 25 janvier au 28 février et touchant à divers degrés la France et une large partie de l'Europe (MEDD, 2002). Cette très forte instabilité a provoqué à plusieurs reprises des 


\section{XIII ${ }^{\text {èmes }}$ Journées Nationales Génie Côtier - Génie Civil \\ Dunkerque, 2-4 juillet 2014}

conditions de houles particulièrement intenses ayant affectées l'ensemble des littoraux atlantique et Manche, E17, E18, E19, E20 (tableau 1). Lors de l'évènement E1825/01/1990 (figure 2), associé à la circulation de dépression nommée Daria, les littoraux principalement affectés par les vagues sont les côtes de Bretagne Nord, de Normandie, de Picardie et tout particulièrement le littoral du Nord Pas de Calais $(\operatorname{Tr}(\mathrm{Hs})=107$ ans).

\subsection{Les évènements récents}

Lors du mois de décembre 1999, deux tempêtes (Lothar et Martin) ont affecté l'ensemble du territoire français à deux jours d'intervalle. Ces évènements ont occasionné des dégâts considérables. Si ces tempêtes ont fortement marqué les esprits, la hauteur des vagues associées à la circulation de ces deux systèmes météorologiques était relativement moyenne (figure 3). Les périodes de retour estimées des hauteurs des vagues étaient au maximum biannuelles en Manche (Lothar) et légèrement inférieure à 20 ans sur la côte Aquitaine (Martin).
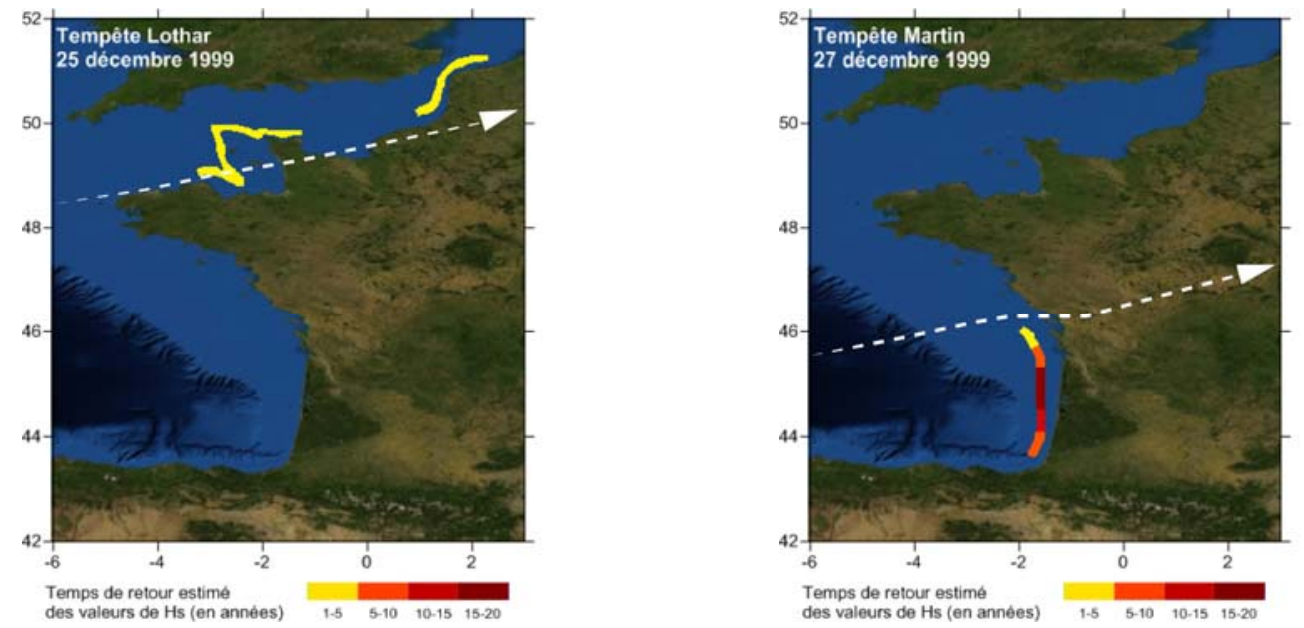

Figure 4. Emprises et période de retour $\operatorname{Tr}(\mathrm{Hs})$ des vagues associés aux évènements météorologiques Martin et Lothar en déc. 1999. Les flèches en pointillés représentent les trajectoires schématiques des centres dépressionnaires, d'après MEDD (2002).

Un exemple significatif d'un évènement météorologique extrême associé à des vagues moyennes est la tempête de 1987, déclaré cyclone du siècle, MEDD (2002). Ce système est responsable du record de pression au niveau de la mer mesuré en France : $948 \mathrm{hPa}$, le 16 Octobre 1987 à Ouessant. Pour autant, les vagues associées à cet évènement sont, selon nos résultats (E14, tableau 1), inférieures aux valeurs décennales (à l'exception du Nord Pas de Calais). C'est principalement la trajectoire atypique de ce système météorologique (trajectoire Sud/Nord du large de la Galice vers le sud de l'Angleterre en passant au large de la Bretagne) qui n’a pas permis le développement de fortes houles mis en part en Manche. Des arguments similaires peuvent être avancés concernant la tempête Xynthia du 28 février 2010 (BERTIN et al., 2012). Bien que cet 


\section{Thème 1 - Hydrodynamique côtière}

évènement soit hors de la période couverte par la base de données BoBWA-10kH, on peut observer que durant cette tempête, les conditions de vagues enregistrées à la bouée Gascogne (Hs=5,9 m) sont nettement inférieures à un évènement de période de retour annuelle $(\mathrm{Hs}(\operatorname{Tr}=1 \mathrm{an})=10,2 \mathrm{~m})$. La trajectoire de la tempête Xynthia (Sud-Ouest NordEst) est comparable à celle de la tempête d'octobre 1987 et explique pour les mêmes raisons que les vagues aient été de hauteurs modérées.

\section{Discussion}

Dans cette étude, les périodes de retour calculées sont des valeurs théoriques basées sur les lois statistiques obtenues lors de la création de la base de données BoBWA-X. Par ailleurs, les données sur lesquelles reposent les statistiques sont des modélisations rétrospectives (base de données BoBWA-10kH) ne prenant pas en compte les variations de niveau d'eau (marée, surcotes) et les courants (tout comme les autres simulations rétrospectives disponibles sur de telles périodes pluri-décennales). Pour ces raisons, la base de données BoBWA-10kH doit a priori être utilisée avec précaution (principalement en Manche). Enfin, la base de données est limitée dans le temps jusqu’à août 2002, aussi les évènements les plus récents (ex: Klaus 24/01/09, Xynthia 28/02/10) n'ont pu être pris en compte lors de l'analyse statistique des valeurs extrêmes. Toutefois, trois points permettent d'apporter des précisions sur ces limites et d'attribuer aux estimations proposées une grande vraisemblance :

i) la qualité du jeu de données BoBWA 10-kH pour lequel les comparaisons globales, sur les plus forts quantiles (LECACHEUX \& PARIS, 2013), comme sur les fortes houles évènement par évènement (BULTEAU et al. 2013b) présentent de bons résultats.

ii) les critères d'homogénéité de sélection des points d'analyse et l'emploi d'une méthode statistique basée sur la diminution de la subjectivité de l'opérateur, permettent de procéder à des inter-comparaisons entre les points d'analyse en limitant la variabilité des valeurs extrêmes calculées.

iii)Les évènements récents (sur la période 2002-2013) ont été d’intensité relativement modérée et ne dépassent globalement pas les hauteurs de vagues décennales déterminées par BoBWA-X. Par exemple, la bouée Gascogne (Météo-France bouée 62001), sur la période 2002-2014, ne présente qu'une seule mesure supérieure à $11 \mathrm{~m}$ ( $\operatorname{Tr}(\mathrm{Hs}=11 \mathrm{~m}) \sim 1-2$ ans selon BoBWA-X). Il s'agit de l'évènement de forte houle associé à la dépression Hercule (5-7 janvier 2014) caractérisé par des valeurs de 11,9 $m$ soit un évènement de période de retour comprise entre 4 et 5 ans. De même, en Manche, sur la période de données disponibles de 2002 à 2009 à la bouée Minquiers, aucune mesure de Hs n’a dépassée 5,6 m, CETMEF (2012). Selon nos résultats, cette valeur a une période de retour théorique de l'ordre de 7 ans.

On peut donc considérer qu'au regard des observations disponibles, l’intégration des évènements de fortes houles des 10 dernières années n’aurait que des conséquences 


\section{XIII ${ }^{\text {èmes }}$ Journées Nationales Génie Côtier - Génie Civil \\ Dunkerque, 2-4 juillet 2014}

marginales sur les valeurs extrêmes fournies dans BoBWA-X et ne modifierait donc pas l’ordre de grandeur des périodes de retours $\operatorname{Tr}(\mathrm{Hs})$ des évènements passés présentés. Ce point reste toutefois à préciser pour les secteurs de la côte directement impactés lors d'événements récents. Notamment, la tempête Klaus (janvier 2009), qui a affectée la Gironde et les Landes. Les mesures locales de hauteurs significatives à la bouée Cap Ferret ont atteint 11,3 m (CETMEF, 2012), valeur comprise dans l'intervalle de confiance 95\% de la valeur centennale déterminée dans BoBWA-X pour ce point. Elle aurait donc un impact non négligeable sur des statistiques d'extrêmes locaux intégrant cet évènement.

\section{Conclusion}

L'utilisation conjointe de la base de données BoBWA-10kH (séries temporelle de vagues) et de la base de données statistique des extrêmes de vague (BoBWA-X), sur la période 1958-2002 a permis d’identifier les évènements de fortes houles les plus intenses (de période de retour $\operatorname{Tr}(\mathrm{Hs})$ supérieur à 10 ans) le long des façades métropolitaines atlantique et Manche. Ces évènements ont été analysés par rapport aux périodes de retour local des pics de vague et à l'extension du linéaire côtier concerné. Parmi les évènements identifiés, on note: le 21/01/1965, $\operatorname{Tr}(\mathrm{Hs})_{\max }=77$ ans (Côte d'Armor) ; le 02/12/1976, $\operatorname{Tr}(\mathrm{Hs})_{\max }=80$ ans (Gironde) ; le 25/01/1990, $\operatorname{Tr}(\mathrm{Hs})_{\max }=107$ ans (Nord Pas de Calais) ou encore le 12/02/1990, $\operatorname{Tr}(\mathrm{Hs})_{\max }=92$ ans (Finistère). Ce travail illustre notamment que des tempêtes marquantes (Lothar, Martin, Xynthia) ne sont pas forcément associées à de fortes vagues. A l'inverse, un évènement de forte houle n'est pas nécessairement synonyme d'impacts importants à la côte en termes d'érosion ou submersion. Une analyse approfondie des concomitances entre marée, surcotes et houles permettrait d'identifier les contextes dans lesquelles les évènements de fortes houles recensés ont eu lieu et ainsi mieux envisager des scénarios extrêmes plausibles pour les littoraux métropolitains.

Par ailleurs, comme illustré par les évènements érosifs entre décembre 2013 et février 2014, il convient d'approfondir l'analyse des caractéristiques (durée, hauteurs significative, longueurs d'onde, directions des vagues) et de la récurrence, des évènements de houles fortes à modérées.

\section{Remerciements :}

Les auteurs remercient Elodie Charles pour l'utilisation de la base de données de vagues BoBWA 10-kH, issue de son travail de thèse (financé par les Fonds AXA pour la recherche) réalisé dans le cadre d'une collaboration associant le BRGM et CNRMGAME (Météo-France - CNRS). Nous remercions également la DGPR du Ministère de l'Ecologie, du Développement Durable et de l'Energie pour le co-financement de l'étude climat de houle II ayant permis de réalisé cette étude. Enfin nous remercions François Paris pour ses remarques et ses apports lors du projet Climat de houle I. 


\section{Références bibliographiques}

BENOIT M., LAFON F., GOASGUEN G. (2006). Constitution et exploitation d'une base de données d'états de mer le long des côtes Françaises par simulation numérique sur 23 ans. IXèmes Journées Nationales Génie Côtier Génie Civil, Brest (France), Septembre 12-14, pp 21-30. http://dx.doi.org/10.5150/ingcgc.2006.003-B

BERTIN X., LI K., ROLAND A., BREILH J.F., CHAUMILLON E. (2012). Contributions des vagues dans la surcote associée à la tempête Xynthia, février 2010. XIIèmes Journées Nationales Génie Côtier - Génie Civil, Cherbourg, 12-14 juin 2012 http://dx.doi.org/10.5150/ingcgc.2012.099-B

CETMEF (2012). Fiches synthétiques de mesure des états de mer. Centre d'Etudes Techniques Maritimes et Fluviales, février 2012.

BESSEMOULIN P. (2002). Les tempêtes en France. Les annales des mines, réalités industrielles, août 2002, 6 p.

BULTEAU T., NICOLAE LERMA A., LECACHEUX S., PARIS F. (2013a). Spatial Extreme Value Analysis of Significant Wave Heights Along the French Coast. in Actes from International Short Conference on Extreme Value Analysis and Application to Natural Hazards (EVAN2013), Siegen, 8-11 September, 10 p.

BULTEAU T., NICOLAE LERMA A., LECACHEUX S. (2013b). Projet Climats de houle - Phase 2 : Analyse des valeurs extrêmes de vagues le long de la côte atlantique et en Manche. Rapport final. BRGM/RP-62730-FR, 49 p., 26 fig., 3 ann. http://infoterre.brgm.fr/rapports/RP-62730-FR.pdf.

CHARLES, E., IDIER, D., THIÉBOT, J., LE COZANNET, G., PEDREROS, R., ARDHUIN, F. AND PLANTON, S. (2012). Present Wave Climate in the Bay of Biscay: Spatiotemporal Variability and Trends from 1958 to 2001. Journal of Climate, Vol. 25 (6), pp 2020-2039. http://dx.doi.org/10.1175/JCLI-D-11-00086.1

MEDD (2002). Les tempêtes, risques naturels majeurs. Dossier d'information ministère de l’Écologie et du Développement durable, 28 p.

LECACHEUX S., PARIS F. (2013). Projet Climats de houle - phase 1 : Intercomparaison de simulations rétrospectives et prospectives dans le Golfe de Gascogne. Rapport BRGM/RP-61651-FR, $116 \quad$ p., 58 ill, 1 ann. http://infoterre.brgm.fr/rapports/RP-61651-FR.pdf.

MAZAS F., HAMM L. (2011). A multi-distribution approach to POT methods for determining extreme wave heights. Coastal Engineering. Vol. 58, pp 385-394. http://dx.doi.org/10.1016/i.coastaleng.2010.12.003

THOMPSON P., CAI Y., REEVE D., STANDER J. (2009). Automated threshold selection methods for extreme wave analysis. Coastal Eng. Vol. 56, pp 1013-1021. http://dx.doi.org/10.1016/j.coastaleng.2009.06.003 